Pteridines

Vol. 13, 2002, pp. 1 - 8

\title{
Urinary Neopterin Indicates Early Infection and Disease Progression: Model Studies with Simian and Human Immunodeficiency Viruses in Macaques
}

\author{
Christiane Stahl-Hennig, ' Claudia Fendrich,' Wolfgang Lüke,' Bernhard Widner, ${ }^{2}$ Gerhard Hunsmann', \\ Dietmar Fuchs ${ }^{2}$
}

'German Primate Centre, Division of Virology and Immunology, Göttingen, Germany, ${ }^{2}$ Institute of Medical Chemistry and Biochemistry, University of Innsbruck, and Ludwig-Boltzmann-Institute for AIDS-Research, A-6020 Innsbruck, Austria

\begin{abstract}
The value of urinary neopterin as a predictive marker for disease progression in SIV- and HIV-2-infected rhesus (Macaca mulatta) and cynomolgus macaques (Macaca fascicularis) was assessed by comparing pre- with postinfection data. Before infection stable baselines for neopterin were observed in both species with significantly higher concentrations in cynomolgus macaques than in rhesus macaques. After infection of cynomolgus macaques with human immunodeficiency virus type 2 (HIV-2) neopterin concentrations exceeded $1.2-4.2$ times preinfection values, whereas rhesus monkeys infected with simian immunodeficiency virus (SIV) yielded concentrations of more than 8 times above baseline. Increased neopterin concentrations always preceded seroconversion. No rise of neopterin was observed in cynomolgus macaques remaining seronegative after inoculation with HIV-2, whereas after inoculation with SIV neopterin was slightly elevated in rhesus monkeys despite remaining seronegative. In animals with high virus replication a pronounced increase of neopterin levels was followed by signs of immunodeficiency. Therefore like in HIV-1-infected man, in macaques infected with SIV or HIV-2 the urinary neopterin concentration is an early and reliably predictive marker for AIDS disease progression and reflects pathogenicity. This parameter can be easily assessed in study protocols for drug and vaccine tests in monkeys as in man.
\end{abstract}

Key words: Virus Isolation, Seroconversion, Animal Model, AIDS, Neopterin, SIV, HIV-2, macaques

\section{Introduction}

Several serological and immunological parameters have been investigated in the early and late stages of HIV-infection and have been assessed as predictive marker for disease progression. In the later stages of the infection, the most prominent change is a massive CD4+ cell depletion leading to reduced $\mathrm{CD} 4+/ \mathrm{CD} 8+$ cell ratios (1). These parameters are inversely correlated to serum B2-microglobulin (B2M) (2) and neopterin (3-5) concentrations. HIV activity can be directly determined by measuring serum p24 antigen concentrations, but this antigen is not present in all patients and its reproducible quantitative determination is problematic (6). Also measurement of acid-dissociated p24 antigen has its limitations when compared to other predictive parameters in the course of HIV infection (7). To date direct quantification of HIV load by poly- merase chain reaction is the method of choice for follow-up therapy success in HIV infected individuals (8).

For the very early phase of HIV-infection, the socalled primary HIV-infection (9), changes in unspecific immune activation markers such as serum neopterin and B2M (9) have been described $(10,11)$. These parameters are probably interrelated. In contrast to most other markers of immune activation neopterin can also be measured in the urine (12) which represents a simple, non-invasive method to monitor alterations of the immune system even in the early stages of HIV-infection.

The experimental infection of macaques with human $(13,14)$ or simian immunodeficiency viruses (14) represent valuable animal models for AIDS research. In a preliminary study involving two rhesus monkeys, we could demonstrate that the increase in

Correspondence to: Dr. C. Stahl-Hennig, Deutsches Primatenzentrum, Kellnerweg 4, D-37077 Göttingen, Germany, Phone: ++49 551-3851-154, Telefax: ++49 551-3851-184 e.mail: stahlh@www.dpz.gwdg.de 
morning urinary neopterin concentration was a very early marker for the productive infection with simian immunodeficiency virus (SIV) isolate SIVmac251 (15). Similar results have been described for serum neopterin in SIV-infected (16) and for CSF in D-retrovirus-infected rhesus monkeys (17). In addition to SIVmac, macaques can be infected experimentally with other SIV isolates (14) and with $\operatorname{HIV}-2(13,14)$. In this study, urinary neopterin concentrations were systematically evaluated in 14 rhesus and 12 cynomolgus macaques infected with undiluted or serial dilution of either SIVagm, SIVmac or HIV-2ben.

\section{Materials and Methods}

14 rhesus monkeys (Macaca mulatta) of Chinese or Indian origin and 12 cynomolgus macaques (Macaca fascicularis) originating from the Philippines or Mauritius Island were experimentally infected. At the time of infection all monkeys were about $2-2.5$ years old and seronegative for STLV-1, SIV, and D-type retrovirus. Animal husbandry, blood and urine sampling and determination of neopterin have been described (15). Urine samples were stored at $-20^{\circ} \mathrm{C} 2$. 4 hours after collection and sent at ambient temperature without further addition of preservatives once a week by regular mail to the investigating laboratory. Preinfection neopterin concentrations in morning urine samples were determined in relation to creatinine concentrations. Neopterin concentrations after infection were expressed as a percent of the individual mean levels before inoculation.

Virus isolates and their dilutions used to infect the two macaque species are shown in Tab. 1. All monkeys were inoculated intravenously either with cell-free virus taken directly from culture supernatant fluid or with dilutions of this material each in RPMI 1640 supplemented with $10 \%$ fetal calf serum.

SIVagmTYO-1 had been passaged once in vivo prior to infection of two rhesus macaques. This virus was isolated from rhesus monkey \#5264 tcn weeks after infection (18). The original SIVmac251 (19) had also been passaged once in a rhesus monkey. This reisolate was designated SIVmac32H (20) and grown on C8166 cells. Six rhesus monkeys were injected with one $\mathrm{mL}$ of a $10^{-2}$ dilution and two animals each with one $\mathrm{mL}$ of either a $10^{-4}$ or $10^{-6}$ dilution of this virus stock. HIV-2ben-infection of macaques has recently been described (21). Four monkeys from this earlier experiment, two rhesus and two cynomolgus macaques infected with an undiluted stock virus of HIV-2ben (stock 1), were also included in the present study. In order to determine the monkey infectious dose 50 (MID50) of a different, newly prepared HIV-2ben virus stock (stock 2) intended to be used as challenge virus for future vaccine experiments and grown on Molt- 4 clone 8 cells, three cynomolgus monkeys each received $1 \mathrm{~mL}$ of a $10^{-4}$ or $10^{-5}$ dilution and two animals $1 \mathrm{~mL}$ of a $10^{-6}$ dilution. These dilutions were chosen according to the in vitro titer of $104.5 \mathrm{TCID} 50 / \mathrm{mL}$ determined on MOLT- 4 clone 8 cells. Two other cynomolgus macaques were inoculated with the undiluted stock virus derived from the biologically active molecular clone MK6 of HIV-2ben (22). SIVagmTYO-1 and SIVmac were isolated by cocultivation of Molt- 4 clone 8 or C81-66 cells, respectively, with peripheral blood mononuclear cells (PBMC) of the inoculated monkeys. HIV-2ben reisolation and monitoring of seroconversion and immunological and clinical changes have been reported (21).

Grouped data were statistically evaluated with the Mann Whitney test. Neopterin concentrations were compared with preinfectious levels using Wilcoxon matched pairs signed rank test. Correlation was analysed by Spearman's rank correlation coefficients.

\section{Results}

Preinfection urinary neopterin concentrations: In both macaque species stable baselines for neopterin were measured with an interindividual variation of 20 $50 \%$. The mean physiological neopterin concentrations in cynomolgus macaques of 259.3 (S.D. \pm 132.7 $\mu \mathrm{mol} / \mathrm{mol}$ creatinine) were more than two times higher than concentrations found in rhesus monkeys (115.4 \pm $38.6 \mu \mathrm{mol} / \mathrm{mol}$ creatinine; $\mathrm{p}<0.01)$.

Virus isolation and seroconversion: In (Tab. 1) 1 rhesus and cynomolgus monkeys involved in this study; the respective virus strains used for inoculation; the corresponding virus dilutions injected and the outcome of infection are listed. All rhesus monkeys infected either with SIVagmTYO-1 or with SIVmac at a dilution of $10^{-2}$ and one of two animals which received SIVmac diluted to $10^{-4}$ seroconverted within 4 to 6 weeks after infection. The other rhesus monkey receiving SIVmac diluted to $10^{-4}$ and both animals injected with SIVmac diluted to $10^{-6}$ remained seronegative. One rhesus monkey inoculated with undiluted HIV-2ben (stock 1) and all cynomolgus macaques inoculated with either undiluted uncloned (stock 1) or clone-derived HIV-2ben, as well as those inoculated with $10^{-4}$ of the new uncloned HIV-2ben stock (stock 2) seroconverted within 4 weeks after infection. All cynomolgus monkeys inoculated with $10^{-5}$ or $10^{-6}$ of the HIV-2ben virus stock 2 did not seroconvert. Virus was reisolated within six weeks after infection (Tab. 1) from all seropositive cynomolgus macaques except for one animal infected with the clone-derived virus. In 
Table 1: Response of macaques to infection with SIV or HIV-2

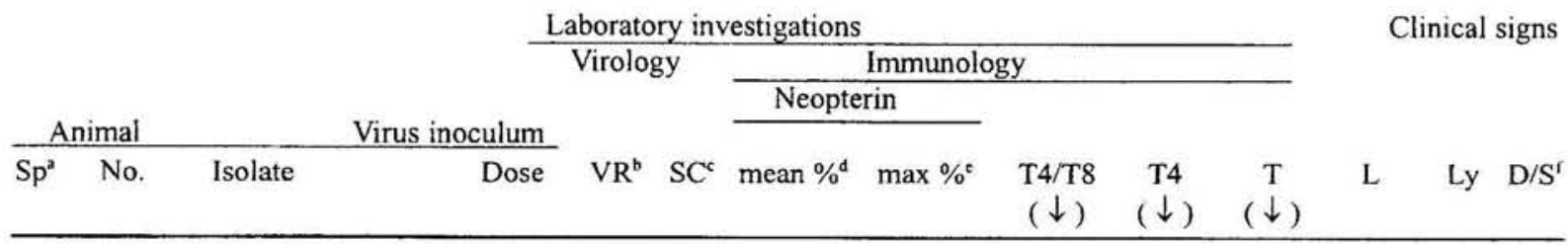

\begin{tabular}{lll}
\multicolumn{3}{l}{ A: Seropositive macaques } \\
MM & 5025 & SIVagmTYO-1 \\
MM & 5026 & SIVagmTYO-1 \\
MM & 1689 & SIVmac251/32H \\
MM & 1705 & SIVmac251/32H \\
MM & 5024 & SIVmac251/32H \\
MM & 5028 & SIVmac251/32H \\
MM & 5129 & SIVmac251/32H \\
MM & 5133 & SIVmac251/32H \\
MM & 5019 & SIVmac251/32H \\
MM & 5020 & HIV-2ben \\
MF & 5172 & HIV-2ben \\
MF & 5173 & HIV-2ben \\
MF & 5344 & HIV-2ben \\
MF & 5345 & HIV-2ben \\
MF & 5346 & HIV-2ben \\
MF & 5170 & HIV-2benMK6 \\
MF & 5171 & HIV-2benMK6
\end{tabular}

$\begin{array}{rrrrr}1 & - & 6 & 365 & 1855 \\ 1 & 4 & 6 & 249 & 2248 \\ 10^{-2} & \geq 2 & 4 & 305 & 800 \\ 10^{-2} & \geq 2 & 4 & 373 & 869 \\ 10^{-2} & \geq 4 & 4 & 620 & 2917 \\ 10^{-2} & \geq 2 & 4 & 700 & 2162 \\ 10^{-2} & \geq 2 & 4 & 450 & 1571 \\ 10^{-2} & \geq 2 & 4 & 490 & 1505 \\ 10^{-4} & \geq 4 & 4 & 712 & 3739 \\ 1 & - & 4 & 192 & 405 \\ 1 & 4-20 & 4 & 200 & 392 \\ 1 & 2-20 & 4 & 106 & 178 \\ 10^{-4} & 2-32 & 4 & 92 & 121 \\ 10^{-4} & \geq 4 & 4 & 148 & 293 \\ 10^{-4} & 2-24 & 4 & 98 & 194 \\ 1 & - & 4 & 91 & 130 \\ 1 & 2-4 & 4 & 225 & 418\end{array}$

\section{B: Seropositive macaques}

\begin{tabular}{|c|c|c|c|c|c|c|c|c|c|c|c|c|}
\hline $\mathrm{MM}$ & 5018 & SIVmac & $10^{-4}$ & - & - & 136 & 260 & - & - & - & - & - \\
\hline MM & 5022 & SIVmac & $10^{-6}$ & - & - & 110 & 477 & - & - & - & - & - \\
\hline MM & 5023 & SIVmac & $10^{-6}$ & - & - & 122 & 176 & - & - & - & - & - \\
\hline MM & 5021 & HIV-2ben & 1 & - & - & 201 & 384 & - & - & - & - & - \\
\hline $\mathrm{MM}$ & 5348 & HIV-2ben & $10^{-5}$ & - & - & 81 & 98 & - & - & - & - & - \\
\hline MM & 5353 & HIV-2ben & $10^{-5}$ & - & - & 101 & 176 & - & - & - & - & - \\
\hline $\mathrm{MM}$ & 5355 & HIV-2ben & $10^{-5}$ & - & - & 91 & 181 & - & - & - & - & - \\
\hline MM & 5357 & HIV-2ben & $10^{-6}$ & - & - & 83 & 140 & - & - & - & - & - \\
\hline MM & 5358 & HIV-2ben & $10^{-6}$ & - & - & 76 & 128 & - & - & - & - & - \\
\hline
\end{tabular}

'Sp, species; MM, Macaca mulatta; MF, Macaca fascicularis

${ }^{b} \mathrm{VR}$, virus recovery (weeks after infection)

${ }^{\circ} \mathrm{SC}$, seroconversion (weeksafter infection)

${ }^{d}$ mean $\%$, mean neopterin concentration within 40 days after inoculation ( $\%$ of baseline)

' max \%, maximum neopterin concentration after inoculation ( $\%$ of baseline)

${ }^{\prime} \mathrm{T} 4 / \mathrm{T} 8, \mathrm{~T} 4 / \mathrm{T} 8$ cell ratio; $\downarrow$, decrease of; T4, absolute T4 cells; L, lymphadenopathy; T, thrombocytopenia; $\downarrow$, decrease of; abs, absolute; Ly, lymphoma; D, death; S, sacrificed

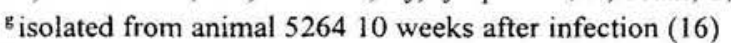

${ }^{\text {h }}$ cell cultured expanded molecular clone of HIV-2ben

contrast to both SIVagm-infected monkeys, from which only one was virus positive at one occasion, virus was reisolated from all SIVmac-infected monkeys at each attempt. Despite seroconversion, virus could neither be recovered from one HIV-2-infected rhesus monkey during the whole observation period. In the majority of the animals cell-associated viraemia preceded seroconversion. Virus could not be recovered from any inoculated monkey remaining seronegative.

Neopterin as a very early response to the infection: In seropositive animals the mean values of the neopterin concentrations measured within 40 days after infection ranged from $91 \%$ to $712 \%$ of the baseline. The maximum values ranged from $121 \%$ to $3739 \%$ (Tab. 1). In seronegative monkeys mean values ranged from $76 \%$ to $201 \%$ and maximum increas- 

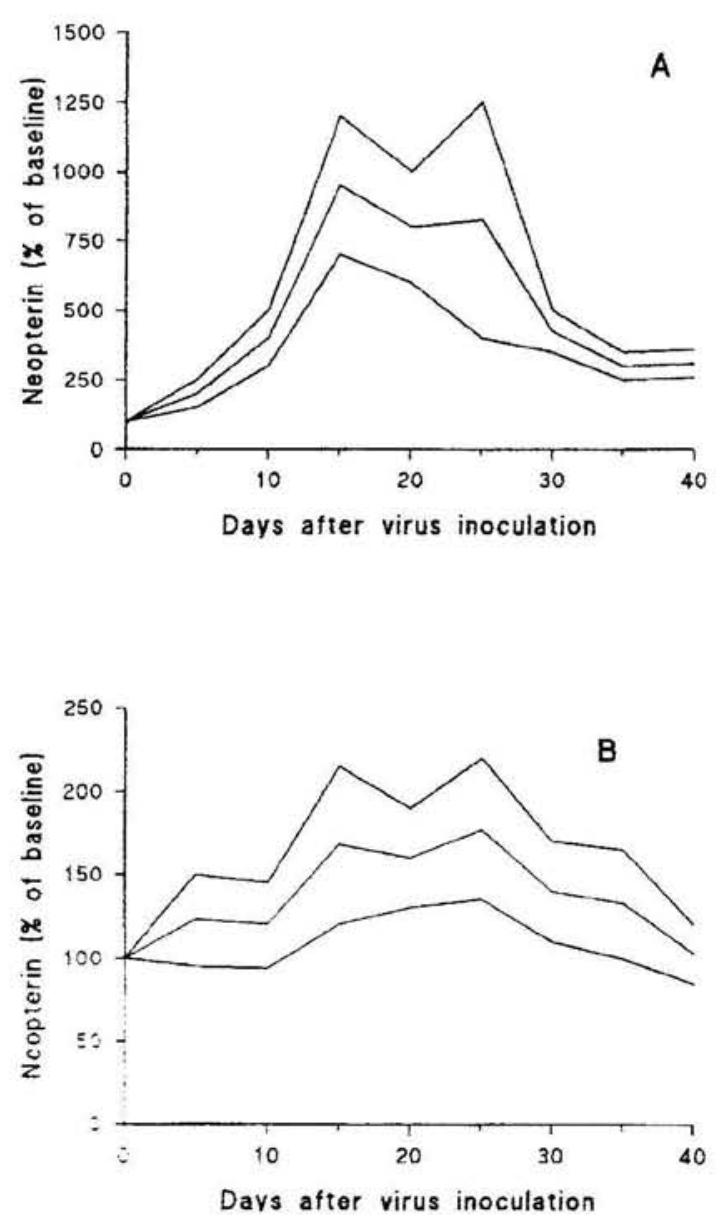

Figure 1: Course of urinary neopterin concentrations (mean \pm S.E.M.) in rhesus and cynomolgus monkeys after inoculation with SIV or HIV-2. Neopterin concentrations obtained after inoculation were related to preinoculation values and expressed as percent of mean baseline values. The diagram shows the mean neopterin concentrations of (A) rhesus monkeys after infection with SIV ( $\mathrm{n}=9$; animal No. 5025, 5026, 1689, $1705,5024,5028,5129,5133,5019$ from Table 1) and of (B) cynomolgus monkeys after infection with HIV2 ben ( $\mathrm{n}=7$; animal No. 5172, 5173, 5344, 5345, 5346, 5170,5171 from Table 1).

cs from $98 \%$ to $477 \%$. No significant differences were found between the preinfection neopterin concentrations of seroconverted and non-seroconverted animals.

The course of mean neopterin concentrations of rhesus monkeys inoculated with SIV is shown in Fig.1A. Average neopterin concentrations were increased tenfold. In 8 of 9 SIV-seropositive monkeys neopterin concentrations were already increased over baseline levels between days 6 to 10 aftcr infection ( $p$ -0.013 , Wilcoxon paired rank test). 11 to 15 days after infection, all SIV positive monkeys showed peak neopterin levels which were dropping thereafter but remained elevated at least three times above the baseline until the end of the 40-days observation period $(\mathrm{p}<0.01)$. In contrast, cynomolgus macaques infected with HIV-2 (Fig.1B) exhibited only mean values two times above preinfection values. In all these monkeys the urinary neopterin concentrations were increased significantly from days 15 to 20 after infection. Concentrations had already declined to baseline levels at the end of the study. In the two rhesus monkeys (\# 5020 and \# 5021) inoculated with undiluted HIV-2 (stock 1) also a weak increase like in the HIV-2-infected cynomolgus monkeys was monitored exceeding the baseline values only up to four times (Tab. 1).

Three rhesus monkeys inoculated with the higher dilutions of SIV mac remained seronegative. The mean neopterin concentrations of this group after inoculation are shown in Fig.2A demonstrating a mean increase of two times of the baseline level. One rhesus monkey receiving the 10-6 virus dilution (\# 5022) showed a significant increase between days 15 to 20 postinoculation, whereas the other two macaques either inoculated with 10-4 (\# 5018) or 10-6 (\# 5023) showed only slight increases of their neopterin concentrations (Tab. 1). In contrast, in cynomolgus monkeys remaining virus and antibody negative after HIV-2ben inoculation the urinary neopterin concentrations were not elevated (Fig.2B). For HIV-2ben-infected monkeys the virus dose correlated significantly with neopterin concentrations measured postinfection (mean in \% baseline: $r s=0.748, p<0.01$, Spearman rank correlation).

Rise of neopterin precedes clinical disease progression: All monkeys which had seroconverted after inoculation with SIVmac $32 \mathrm{H}$ developed a persistent lymphadenopathy of their peripheral lymph nodes. Furthermore, in all these animals a decrease of the $\mathrm{CD} 4+/ \mathrm{CD} 8+$ ratio and of the absolute $\mathrm{CD} 4$ - counts (except \# 5024) was noted following infection (Tab. 1). Blastogenic responses of the PBMC from these infected macaques to phytohemagglutinin (PHA) or concanavalin A were also impaired in comparison with preinfection values and negative controls (data not shown). A severe thrombocytopenia occured as early as 12 weeks after infection in rhesus monkeys of Indian origin (\# 1689 and \# 1705) and in nearly all the others originating from China approximately 3-6 months later. Two monkeys of the SIVmac $32 \mathrm{H}$ group have died from AIDS, one 8 months after infection showing multiple lymphomas (\# 1705), the other 15 months postinoculation presented with generalized lymphadenopathy. Another monkey (\# 1689) developed severe multiple lymphomas and had to be 

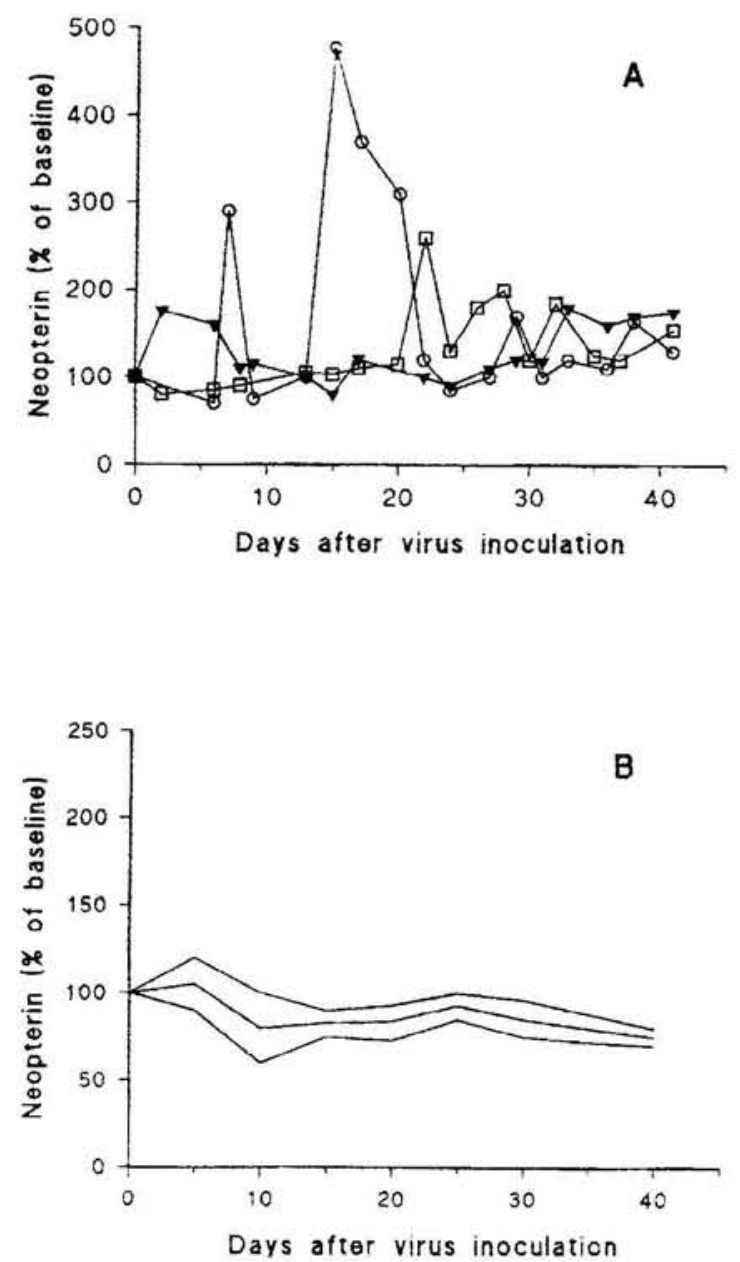

Figure 2: Course of urinary neopterin concentrations (mean \pm S.E.M.) in rhesus and cynomolgus monkeys after inoculation with SIV or HIV-2. Neopterin concentrations obtained after inoculation were related to preinoculation values and expressed as percent of mean baseline values. The diagram shows the mean neopterin concentrations of (A) rhesus monkeys remaining seronegative after inoculation with SIV $(n=3$, animal No. 5018 - open squares, 5022 - open circles, 5023 - filled triangles - from Table l) and of (B) cynomolgus monkeys remaining seronegative after inoculation with HIV-2 ( $\mathrm{n}=5$; animal No. 5348, 5353, $5355,5357,5358$ from Table 1). Note the scale difference on the $\mathrm{Y}$-axes.

euthanised 11 months after infection. In contrast, the two rhesus macaques receiving the in vivo passage of SIVagmTYO-1 and one rhesus monkey and all cynomolgus monkeys infected with clone-derived or uncloned HIV-2ben remained apparently healthy through 14 to 16 months after inoculation. In general, a higher increase of neopterin always preceded clinical changes and alterations of blood parameters indicating immunodeficiency. In the monkeys infected with SIVagmTYO-1 and remaining asymptomatic neopterin appeared to reach the baseline level faster, whereas in the SIVmac-infected macaques showing signs of immunodeficiency neopterin remained on an elevated level during the observation period (data not shown).

\section{Discussion}

In both macaque species stable baseline values of urinary neopterin concentrations were noted for each individuum before infection. Significantly lower physiological neopterin concentrations were observed in rhesus monkeys compared to cynomolgus macaques. Seroconversion after cxpcrimental infection of rhesus and cynomolgus macaques could be demonstrated within 6 weeks after infection. For both, the SIVmac $251 / 32 \mathrm{H}$ and the HIV-2ben virus stock, a very similar in vivo titer could be found, confirming for SIVmac251/32H the endpoint already described $(20,23)$.

Due to infection of rhesus and cynomolgus macaques with SIV and HIV-2, respectively, neopterin concentrations increased significantly and always preceded seroconversion. The increase during the acute phase of infection corresponds well with cytokine mRNA expression in mononuclear cells during acute SIV mac251 infection of macaques (24).

Interestingly, in all SIV-infected rhesus macaques the mean neopterin concentrations increased 12-times over baseline level within 11-15 days after infection. In HIV-2 seropositive cynomolgus macaques the increase was somewhat delayed and only threefold. These data raise the question whether this distinct response is due to the macaque species inoculated or to biological properties of the viruses. Two further observations support the latter explanation. Firstly, in one cynomolgus monkey infected with $1 \mathrm{~mL}$ of a $10^{-2}$ of SIVmac251/32H the neopterin concentrations were still elevated four times above the baseline level 30 to 40 days after infection (data not shown). In contrast, at the same time point neopterin concentrations in all HIV-2ben-infected cynomolgus monkeys were no longer significantly elevated. Secondly, the neopterin concentrations in the one HIV-2ben-infected rhesus monkey (Tab. 1) resemble those reported earlier for two rhesus monkeys infected with SIVmac251 which in our hands was only low pathogenic (15). Thus, the apathogenic HIV-2ben as well as the low pathogenic SIVmac isolate only induced a weak immune activation. This is indicated by a comparably low and shortterm increase of neopterin in both monkey species. In contrast, the pathogenic SIVmac251/32H with a high replicative capacity in vivo as indicated by persistent 
cell-associated viraemia (Tab. 1) led to a higher and faster increase of neopterin remaining elevated even 40 days post-infection. These data reflect the situation observed in HIV-1-infected humans (25). Data on neopterin increase in CSF, but not in serum of D-type retrovirus infected rhesus monkeys (17) further support our assertion that the differences in the elevation of the neopterin levels are virus related.

However, in addition to the virus isolate-dependent increase of neopterin concentrations there also seem to be species-specific reactions. In rhesus monkeys remaining virus and antibody negative after SIVmac inoculation neopterin concentrations increased slightly. Apart from a different immune status of each individual not yet definable, another reason could be the stimulus of a subinfectious viral dose. At present it is not known whether these animals became infected by SIV without measurable seroconversion and virus recovery during the whole follow-up. To clarify the viral status further studies are needed using more sensitive methods such as the polymerase chain reaction (26). On the other hand, according to the in vitro titer of the SIVmac251/32H pool in C $81-66$ cells of 104 TCID $50 / \mathrm{mL}$ (data not shown) used for inoculation it is highly probable that infectious virus had been dilutedout. This obviously unspecific effect was not seen in HIV-2 inoculated seronegative cynomolgus macaques. So far, our data do not allow to conclude whether this phenomenon is due to the immune status of the individual animal or to inherent biological properties of the inoculated virus e.g. infectivity, concentration or replicative efficiency. However, at least a simple antigenic stimulation provided by the fetal calf serum present in the inoculate can be excluded. In six naive rhesus macaques receiving $1 \mathrm{~mL}$ of cell-culture supernatant from non-infected C81-66 cells containing 10\% fetal calf serum no neopterin elevations were measured over 30 days following inoculation (data not shown) thereby supporting our earlier findings (15).

Nevertheless, in all SIVmac251/32H-infected monkeys the high increases in neopterin concentration very early after infection were closely correlated with typical signs of immunodeficiency occurring in later stages of infection. Thus, our findings agree with those described for the early phase of HIV-1-infection in man $(10,11)$, and also an association between a high initial neopterin increase followed by an accelerated loss of CD4 + T-cells has been described in HIV-infected humans (27). Also neopterin determined in various body fluids from HIV-1 seropositive patients has been shown to be a powerful independent predictor of disease progression $(4,28,29)$.

Our findings about the early phase after infection of macaques with SIV or HIV-2 are not only strikingly similar, but moreover exceed data about HIV-1-infection in man. Whereas we could demonstrate a causative relationship between the time point of infection and the occurence of the initial neopterin peak 1014 days after infection, in HIV-1-infected humans the time point of infection very often can only be vaguely estimated retrospectively $(9,10)$. Therefore, elevated neopterin concentrations cannot necessarily be interpreted as a consequence of infection. In these cases one could only speculate about the nature of this correlation, since preinfection values could not be obtained and other acute viral and non-viral infections are also accompanied by high neopterin levels (5).

In our SIV- or HIV-2-infected monkeys urinary neopterin is a very useful adjunct to virus isolation and seroconversion to demonstrate infection because it is sensitive, rises very early and can easily be measured and quantified. Moreover, it predicts disease progression. In addition, this marker coincides with metabolic disorders of the immune system described very recently for SIV-infected macaques (30). Therefore, we suggest to include neopterin as marker in study protocols to monitor therapeutic effects of potential anti-retroviral drugs in SIV- or HIV-2-infected macaques as described in humans $(29,31)$. This marker should also indicate a therapy failure due to the appearance of therapy-resistant mutants. This would enable clinicians to quickly change the therapeutic strategy. Moreover, neopterin is a sensitive and early indicator for infection in experimental vaccine studies (32). Therefore, wc recommend to include this parameter in therapeutic, prophylactic and pathogenesis studies. In recent studies it turned out that the determination of neopterin still has its place even when quantitative HIV RNA measurements with polymerase chain reaction are performed $(8,33)$. Moreover, urine sampling is easier and safer than venipuncture regarding HIV transmissibility (34), and neopterin concentrations remain stable for several days at ambient temperature without additional precautions.

\section{Acknowledgments}

The technical assistance of V. Diaz, C. Oeffner and M. Stöger is gratefully acknowledged. SIVmac $251 / 32 \mathrm{H}$ was kindly provided by Dr. M. Cranage, PHLS Centre for Applied Microbiology and Research, Porton Down, Salisbury, U.K. This work was supported by the Austrian Federal Ministry of Social Affairs and Generations, by the Austrian Funds "Zur Förderung der wissenschaftlichen Forschung", project 14154 Med. as well as by the European Community within the framework of the Concerted Action on "Macaque models for AIDS research". 


\section{References}

1. Nicholson JK, McDougal JS, Jaffe HW, et al. Exposure to human T-lymphotropic virus type III/lymphadenopathy-associated virus and immunologic abnormalities in asymptomatic homosexual man. Ann Intern Med 1985;103:37-42.

2. Hofman B, Wang Y, Cumberland WG, et al. Serum beta2-microglobulin level increases in HIV infection: relation to seroconversion, CD4 T-cell fall and prognosis. AIDS 1990;4:207-214.

3. Fuchs D, Banekovich $M$, Hausen $A$, et al. Neopterin estimation compared with the ratio of Tcell subpopulations in persons infected with human immunodeficiency virus 1. Clin Chem 1988; 34:2415-2417.

4. Fahey JL, Jeremy MD, Taylor PD, et al. The prognostic value of cellular and serological marker in infection with human immunodeficiency virus type 1. N Engl J Med 1990;322:166-172.

5. Fuchs D, Hausen A, Reibnegger G, et al. neopterin as a marker for activated cell-mediated immunity. Immunol Today 1988, 9:150-154.

6. Kruppenbacher JP, Mertens T, Eggers HJ. Reproducibility of HIV antigen test. Lancet 1988; i: 298 .

7. Zaknun D, Orav J, Kornegay J, et al. Correlation of ribonucleic acid polymerase chain reaction, acid dissociated p24 antigen, and neopterin with progression of disease. A retrospective, longitudinal study of vertically acquired human immunodeficiency virus type 1 infection in children. J Pediatr 1997;130:898-905.

8. Salazar Gonzalez JF, Martinez Maza O, Nishanian $P$, et al. Increased immune activation precedes the inflection point of CD4 $\mathrm{T}$ cells and the increased serum virus load in human immunodeficiency virus infection. J Infect Dis 1998;178:423-430.

9. Gaines $H$, von Sydow M, Sönnerborg A, et al. Antibody response in primary human immunodeficiency virus infection. Lancet 1987;i:1249-1254.

10. Gaines H, Von Sydow Mae, Von Stedingk LV, et al. Immunological changes in primary HIV-1 infection. AIDS 1990;4:995-999.

11. Zangerle R, Schönitzer D, Fuchs D, Möst J, Dierich MP, Wachter-H. Reducing HIV transmission by seronegative blood. Lancet 1992; 339:130131.

12. Fuchs D, Werner ER, Wachter H. Soluble products of immune activation: Neopterin. In: Manual of Clinical Laboratory Immunology. 4th edition. Edited by RR Rose, EC deMacario, JL Fahey, H Friedman, GM Penn. Washington, D.C., American Society for Microbiology, 1992, pp 251-255.
13. Letvin N: Animal models for AIDS. Immunol Today 1990;11:322-326

14. Desrosiers RC, Ringler DJ: Use of simian immunodeficiency viruses for AIDS research. Intervirology 1989;30:301-312.

15. Fendrich C, Lüke W, Stahl-Hennig C, et al. Urinary neopterin concentrations in rhesus monkeys after infection with simian immunodeficiency virus (SIVmac251). AIDS 1989;3:305-307.

16. Popov J, Mcgraw T, Hofmann B, et al. Acute lymphoid changes and ongoing immune activation in SIV infection. J Acquir Immune Defic Syndr 1992;5:391-399.

17. Heyes MP, Lackner A, Kaufmann S et al. Cerebrospinal fluid and serum neopterin and biopterin in D-retrovirus-infected rhesus macaques (Macaca mulatta): relationship to clinical and viral status. AIDS 1991;5:555-560.

18. Herchenröder O, Stahl-Hennig C, Lüke W, et al. Experimental infection of rhesus monkeys with SIV isolated from African green monkeys. Intervirology 1989;30 (suppl):66-72.

19. Daniel MD, Letvin NL, King NW, et al. Isolation of T-cell tropic HTLV-III-like retrovirus from macaques. Science 1985;228:1201-1204.

20. Cranage MP, Cook N, Johnstone P, et al. SIV infection of rhesus macaques: in vivo titration of infectivity and development of an experimental vaccine. Eds H Schellekens and MC Horzinek, Animal Models in AIDS, Elsevier Science Publishers BV 1990; pp 103-113.

21. Stahl-Hennig C, Herchenröder O, Nick S, et al. Experimental infection of macaques with HIV2ben, a novel HIV-2 isolate. AIDS 1990;4:611617.

22. Kirchhoff F, Jentsch KD, Bachmann B, et al. A novel proviral clone of HIV-2: biological and phylogenetic relationship to other primate immunodeficiency viruses. Virology 1990;177:305-311.

23. Kitchin PA, Cranage MP, Almond N, et al. Titration of SIVmac 251 ( $32 \mathrm{H}$ isolate) in cynomolgus macaques for use as a challenge in vaccination studies. Eds H Schellekens and MC Horzinek, Animal Models in AIDS, Elsevier Science Publishers BV 1990; pp 115-129.

24. Cheret A, Le Grand-R, Caufour P, et al. Cytokine mRNA expression in mononuclear cells from different tissues during acute SIVmac251 infection of macaques. AIDS Res Hum Retroviruses 1996; 12:1263-1272.

25. Fuchs D, Albert J, Asjö B, et al. Association between serum neopterin concentrations and in vitro replicative capacity of HIV-1 isolates. J Infect Disease 1989;160:724-725. 
26. Kitchin PA, Almond N, Szotyori Z, et al. The use of the polymerase chain reaction for the detection of simian immunodeficiency virus in experimentally infected macaques. J Virol Methods 1990;28:85100.

27. Melmed RN, Taylor JM, Detels R, Bozorgmehri M, Fahey JL. Serum neopterin changes in HIVinfected subjects: indicator of significant pathology, CD4 T cell changes, and the development of AIDS. J Acquir Immune Defic Syndr. 1989;2:7076.

28. Fuchs D, Spira TJ, Hausen A, et al. Neopterin as a predictive marker for disease progression in human immunodeficiency virus type 1 infection. Clin Chem 1989;35:1746-1749.

29. Jacobson MA, Bacchetti P, Kolokathis A, et al. Surrogate markers for survival in patients with AIDS and AIDS related complex treated with zidovudine. Brit Med J 1991;302:73-78.

30. Eck HP, Stahl-Hennig C, Hunsman G, et al. Metabolic disorder as an early consequence of SIVmac251 infection in rhesus macaques (Macaca mulatta). Lancet, Lancet 1991;338:346-347.
31. Hutterer J, Armbruster C, Wallner G, Fuchs D, Vetter N, Wachter H. Early changes of neopterin concentrations during treatment of human immunodeficiency virus infection with zidovudine. J Infect Dis 1992;165:783-784.

32. Stahl-Hennig C, Voss G, Nick S, et al. Tween-ether treated SIV adsorbed to aluminium hydroxyde protects monkeys against experimental SIV-infection. Virology 1992;186:588-596.

33. Zangerle R, Steinhuber S, Sarcletti M, et al. Serum HIV-1 RNA levels compared to soluble markers of immune activation to predict disease progression in HIV-1-infected individuals. Int Arch Allergy Immunol 1998;116:228-239.

34. Skolnik PR, Kosloff BR, Bechtel LJ, et al. Absence of infectious HIV-1 in the urine of seropositive viremic subjects. J Infect Dis 1989;160:1056-1060. 